\title{
Obtención de Isoespintanol por Hidrodestilación y Cristalización a partir del Extracto Bencínico de Oxandra xylopioides
}

\author{
Rubén D. Ramírez, Manuel S. Páez y Alberto A. Angulo* \\ Universidad de Córdoba, Facultad de Ciencias Básicas, Departamento de Química, carrera 6 No. 76-103. \\ Montería, Córdoba-Colombia (e-mail: aanguloo@hotmail.com).
}

${ }^{*}$ Autor a quien debe ser dirigida la correspondencia.

Recibido Mar. 20, 2015; Aceptado May. 28, 2015; Versión final Jul. 24, 2015, Publicado Dic. 2015

\begin{abstract}
Resumen
Esta investigación establece un procedimiento para obtener isoespintanol (IE) por hidrodestilación seguida de cristalización, del extracto bencínico de Oxandra xylopiodes. En la exploración de las condiciones óptimas, se realizó monitoreo por cromatografía en capa delgada, en las distintas etapas del proceso, detectando la presencia de isoespintanol. La proporción de IE en el hidrodestilado se estimó por cromatografía de gases-espectrometría de masas. Los resultados sugieren que la concentración óptima en el proceso de hidrodestilación es $4.54 \mathrm{mg}$ de extracto/mL de agua. Para la extracción con solventes se requieren dos extracciones con $10 \mathrm{~mL}$ de diclorometano por un tiempo de $10 \mathrm{~min}$. El proceso de concentración se fijó a $50^{\circ} \mathrm{C}, 1010 \mathrm{mbar}$ y $80 \mathrm{rpm}$ y la cristalización con $200 \mu \mathrm{L}$ de hexano y un cristal de $10 \mathrm{mg}$ de IE. Se concluye que se puede obtener isoespintanol con $73.12 \%$ de pureza por un método sencillo y económico.
\end{abstract}

Palabras clave: isoespintanol; hidrodestilación; Oxandra xylopioides; volátiles; cromatografía.

\section{Obtainment of Isoespintanol by Hydrodistillation and Crystallization from the Bencinic Extract of Oxandra xylopioides}

\begin{abstract}
This research establishes a procedure to obtain isoespintanol (IE) by means of hydrodistillation followed by crystallization of bencinic extract of Oxandra xylopiodes. In the initial setting of the optimal conditions, monitoring by thin layer chromatography was performed in the different stages of the process, detecting the presence of isoespintanol. The proportion of IE in the hydrodistilled current was estimated by means of gas chromatography-mass spectrometry. Results suggest that the optimal concentration in the process of hydrodistillation is $4.54 \mathrm{mg}$ extract $/ \mathrm{mL}$ of water. For the purposes of the extraction with solvent, two extractions of $10 \mathrm{~mL}$ of dichloromethane for 10 minutes are required. The concentration process was fixed at $50^{\circ} \mathrm{C}, 1010 \mathrm{mbar}$ and $80 \mathrm{rpm}$ and crystallization with $200 \mu \mathrm{L}$ of hexane and one crystal of $10 \mathrm{mg}$ of IE. It is concluded that isoespintanol with a purity of $73.12 \%$ can be obtained using a simple and mot-expensive method.
\end{abstract}

Keywords: isoespintanol; hydrodistillation; Oxandra xylopioides; volatile; chromatography 


\section{INTRODUCCIÓN}

Dentro de las fuentes vegetales de metabolitos activos, se encuentra la familia Annonaceae, constituida por cerca de 130 géneros y aproximadamente 2000 especies (Murillo, 2001). Se ha reportado para el género Oxandra, estudios químicos en 8 de las 26 especies conocidas, y como resultado el aislamiento de compuestos con gran diversidad estructural (Arango et al., 1987a; Arango et al., 1987b; Hocquemiller et al., 1991; Tinto y Blair, 1992; Pino, 2005; Angulo et al, 2007; De Sousa et al., 2014a; De Sousa et al., 2014b.) De Oxandra xylopioides se han aislado el monoterpeno aromático isoespintanol (2-isopropil-3,6-dimetoxi-5metilfenol), el triterpeno cicloartano berenjenol y algunas azafluorenonas (Zhang et al, 1987; Rojano et al., 2007a; Rojano et al., 2007b).

Son diversas las investigaciones desarrolladas en Colombia, en las que se han evaluado las características bioactivas del isoespintanol (IE). Se ha demostrado que el IE y algunos derivados actúan como conservantes en el proceso de oxidación de alimentos (Gil y Rojano, 2012), comparándose su actividad antioxidante con la del reconocido butil hidroxi tolueno (BHT) (Rojano et al., 2008a), en resultados in vitro se demuestra dos veces mayor capacidad antioxidante del IE comparado con su análogo biosintético timol (Rojano, 2008b). En su proyección como antifúngico, se ha encontrado buena respuesta contra la especie Colletotrichum acutatum, patógeno responsable de la antracnosis, enfermedad que ocasiona lesiones ovales de color oscuro en hojas y frutas ocasionando grandes pérdidas económicas (Arango et al, 2007). También posee actividad insecticida frente a Spodoptera frugiperda (gusano cogollero del maíz) (Rojano et al., 2007c). Además, el IE tiene potencial como antiinflamatorio (Rojano et al., 2007a). Se ha demostrado que el bromuro de isoespintanol, induce apoptosis de leucocitos polimorfonucleares, proceso fundamental para el mantenimiento de niveles normales de células circulantes, contribuyendo a la resolución de las respuestas inflamatorias (Dade et al., 2010). En cuanto a la evaluación genotóxica y citotóxica, se ha demostrado el daño de ADN en linfocitos humanos por efecto del IE, mediante la técnica de intercambio de cromátidas hermanas. Los resultados preliminares demuestran que el efecto del isoespintanol sobre los linfocitos humanos no es genotóxico (Muñoz et al., 2008).

El proceso de obtención del IE, no se encuentra tecnificado, pues se sigue la ruta tradicional, a través de extracciones sucesivas sobre el material vegetal con diferentes solventes, fraccionamiento, concentración y purificación empleando técnicas cromatográficas en columna, además de monitoreo continuo por cromatografía en capa delgada (CCD) en cada proceso (Rojano et al., 2007a; García, 2014), demostrando que la investigación y desarrollo de este tipo de materiales terapéuticos, de origen vegetal es una tarea difícil y costosa. Algunos estudios indican que cada nuevo medicamento requiere una inversión de alrededor de US\$100-360 millones y un mínimo de 10 años de trabajo (Rates, 2001).

Investigaciones anteriores sobre 0 . xylopioides realizadas en el laboratorio de Productos Naturales de la Universidad de Córdoba (García, 2014), empleando la ruta del proceso de extracción convencional, han aportado información sobre el método de purificación y las características fisicoquímicas y estructurales del IE, por lo que en la presente investigación, se planteó como objetivo diseñar un procedimiento que permitiera obtener IE con alta pureza, partiendo del extracto bencínico de O. xylopioides, con la mínima inversión de recursos y químicamente amigable. Adicionalmente, éste diseño, puede ser empleado para otras especies de Oxandra, que permita el desarrollo tecnológico de la extracción, aislamiento, purificación y cuantificación de IE.

\section{MATERIALES Y MÉTODOS}

\section{Material vegetal}

Las hojas de O. xylopioides fueron recolectadas de un espécimen ubicado en el Municipio de Montería, Departamento de Córdoba, con las coordenadas $08^{\circ} 48^{\prime} 17^{\prime \prime}$ latitud norte y $75^{\circ} 42^{\prime} 07^{\prime \prime}$ longitud oeste, en septiembre de 2012. Un ejemplar de herbario identificado por el Biólogo Alvaro Cogollo Pacheco, se encuentra depositado en el Jardín Botánico Joaquín Antonio Uribe, con el número de colección JAUM 037849 .

\section{Obtención de extracto bencinico de hojas de O. xylopioides}

Las hojas de $O$. xylopioides libres de impurezas, se secaron por ocho días, se disminuyó su tamaño en un molino casero obteniéndose $4,45 \mathrm{~kg}$. Este material fue sometido a extracción exhaustiva por percolación con $12 \mathrm{~L}$ de etanol al $96 \%$. El extracto etanólico fue concentrado a presión reducida obteniéndose $500 \mathrm{~g}$ de extracto etanólico de las hojas. A continuación $50 \mathrm{~g}$ se sometieron a partición para obtener 17,4 g de extracto bencínico. El procedimiento descrito a continuación, se realizó 5 veces para cada cantidad de isoespintanol. Estudio exploratorio 
Hidrodestilación: Inicialmente, se pesaron réplicas de 20,50 y $100 \mathrm{mg}$ de isoespintanol puro. Posteriormente se disolvieron en $2 \mathrm{~mL}$ de diclorometano (DCM). Cada mezcla se transvasó a un balón de destilación de 25 $\mathrm{mL}$ y se agregaron $15 \mathrm{~mL}$ de agua. Se acopló el montaje de microdestilación y se inició el calentamiento con agitación magnética. Luego se midieron los tiempos para evaporación completa de cada mezcla. El hidrodestilado fue fraccionado y monitoreado por cromatografía en capa delgada (CCD), a fin de observar diferencias en el contenido de isoespintanol respecto a los tiempos de destilación.

Extracción: Los volátiles obtenidos en el proceso anterior, fueron extraídos con volúmenes de DCM de 10 y $15 \mathrm{~mL}$. Se monitoreó la presencia del isoespintanol sobre la fase acuosa por CCD. Así mismo, se midieron tiempos de 5, 10 y 15 minutos para la extracción óptima. El sistema de elusión empleado, fue una mezcla DCM-Bencina (1:1).

Reducción de volumen: Los extractos obtenidos fueron sometidos a rotavaporación en un rotavapor Heidoph Hei-VAP Precision, por un periodo de tiempo en el que se redujo el volumen de 2 a $3 \mathrm{~mL}$. La temperatura del baño fue de $50^{\circ} \mathrm{C}$, presión atmosférica de $1010 \mathrm{mbar}$ y velocidad de rotación de $80 \mathrm{rpm}$. Se realizó monitoreo por CCD del solvente destilado en el balón colector para detectar la presencia de isoespintanol.

Contenido de volátiles: Luego de reducir los volúmenes se procedió a trasvasar los volátiles contenidos en el balón de rotavaporación, a viales previamente pesados y rotulados. Se dejaron secar a temperatura ambiente por un periodo de 1 día. Después se procedió a pesarlos para determinar el rendimiento del proceso de extracción. Se realizó monitoreo por CCD para verificar la presencia del isoespintanol.

Cristalización: A cada vial anterior, se adicionaron $200 \mu \mathrm{L}$ de hexano y enseguida, se sumergió un cristal de IE de masa conocida (de 5 a $10 \mathrm{mg}$ ), que actuó como núcleo acelerando la reacción en cadena que provoca el crecimiento de cristales.

Ensayo con extractos: Las anteriores operaciones, son ejecutadas (5 veces) para el extracto bencínico (EB) a fin de ajustar variables operativas propias de la naturaleza de ésta matriz.

\section{Aplicación de la técnica a muestras de extracto bencínico}

Una vez establecidas las condiciones para el extracto bencínico, se desarrolló el método (hasta reducción del volumen) para tres muestras, con el objetivo de obtener los volátiles y prepararlos para la determinación del contenido de isoespintanol por cromatografía de gases acoplada a espectrometría de masas (CG-EM). Adicionalmente se analizó una muestra de isoespintanol (estándar de 99\% de pureza obtenido por García, 2014) para determinar el tiempo de retención y el espectro de masas para la comparación con las muestras de volátiles.

\section{Caracterización y contenido de IE en los volátiles}

El análisis de composición química, se realizó empleando un cromatógrafo de gases acoplado a un espectrómetro de masas Thermo Scientific modelo Trace 1310, equipado con una columna AB-5MS, de 30 $\mathrm{m}$ de longitud, $0.25 \mathrm{~mm}$ de diámetro interno y $0.25 \mu \mathrm{m}$ espesor de película. Los volátiles $(30 \mathrm{mg})$ se diluyeron en $1 \mathrm{~mL}$ de $\mathrm{DCM}$, luego se tomó $1 \mu \mathrm{L}$ y se disolvió hasta $1 \mathrm{~mL}$. Se ejecutaron las mismas diluciones para el estándar. El sistema de gradientes de temperatura inició con $80^{\circ} \mathrm{C}$ por 10 minutos, luego un aumento hasta $200^{\circ} \mathrm{C}$ a $10^{\circ} \mathrm{C} / \mathrm{min}$. Se incrementó la temperatura hasta $240^{\circ} \mathrm{C}$ a $4^{\circ} \mathrm{C} / \mathrm{min}$ y finalmente se llevó hasta $290^{\circ} \mathrm{C}$ por 10 minutos a $10^{\circ} \mathrm{C} / \mathrm{min}$. La inyección fue tipo splitless, con un volumen de inyección de $1 \mu \mathrm{L}$ en modo de ionización por impacto electrónico a 70 eV.

Para la determinación del contenido de IE se tuvieron en cuenta los porcentajes obtenidos en los cromatogramas de iones totales. Se empleó la normalización de áreas para evaluar los contenidos de IE en las muestras de EB analizadas, en términos de proporción relativa promedio.

\section{Análisis estadístico}

Luego de la identificación de factores de influencia controlable y no controlable y la minimización de sus efectos y buscando optimizar el método, se evaluó el factor principal (concentración del EB) que impacta sobre el rendimiento en la obtención de los volátiles (incluyendo IE). Se procedió considerando cuatro concentraciones del EB, como se aprecia en la Tabla 1.

Se realizó una aleatorización de los tratamientos a ejecutar empleando una tabla de números aleatorios. Una vez definido el orden, se ejecutó el procedimiento para tres réplicas por cada tratamiento, para evaluar la dispersión de los resultados a través de la desviación estándar y evaluar, a través de análisis de varianza 
(ANAVA) de un factor, la significancia estadística $(p<0,05)$ de los tratamientos ensayados sobre el rendimiento global del proceso de obtención de los volátiles. Como requisito para la aplicación de ANAVA paramétrica, se realizó un análisis de valores atípicos y se verificaron los supuestos de normalidad y homogeneidad de varianzas. Se realizó la prueba de Tukey $(p<0,05)$ para evaluar qué tratamientos se relacionan con el mayor porcentaje de los volátiles. El análisis estadístico se desarrolló empleando el paquete estadístico Minitab 17.

Tabla 1. Tratamientos del factor concentración

\begin{tabular}{|c|c|}
\hline Tratamientos & Concentraciones del EB ensayadas $(\mathrm{mg} / \mathrm{mL})$ \\
\hline T1 & 2,27 \\
\hline T2 & 4,54 \\
\hline T3 & 5,88 \\
\hline T4 & 8,33 \\
\hline
\end{tabular}

\section{RESULTADOS Y DISCUSIÓN}

A partir de los resultados de medidas repetidas, obtenidos en el estudio exploratorio, se evaluó la cantidad de EB óptima para la ejecución del diseño experimental. Los mejores resultados en términos de exactitud y precisión se obtuvieron para $100 \mathrm{mg}$ de EB.

\section{Caracterización y contenido de IE en los volátiles}

El análisis cuantitativo de los cromatogramas, evidencia que el componente mayoritario de los volátiles obtenidos fue el IE con un promedio de $73,12 \%$ para las tres muestras analizadas. El tiempo de retención para el IE bajo las condiciones ensayadas fue de 20,10 a 20,12 minutos. Los espectros de masas de las muestras sometidas al proceso de análisis por CG-EM conservan el mismo patrón de fragmentación, que corresponde con el estándar de isoespintanol. En cada espectro se aprecia el pico ion molecular de relación $\mathrm{m} / \mathrm{z} 210$ y la pérdida sucesiva de radicales metilo con fragmentos de relación m/z 195 (100\%), 180, 165, 150, 135. Además, se observa un pico de baja intensidad en $\mathrm{m} / \mathrm{z} 91$ que corresponde al ion tropilio.

\section{Niveles de concentración y rendimiento}

El análisis de cada etapa del proceso permitió ajustar las variables para minimizar las pérdidas de rendimiento en el proceso de extracción y de esta manera optimizarlo (Limón et al. 2012). Se identificó en el estudio exploratorio que la variable con mayor influencia sobre el rendimiento fue la concentración del extracto de partida en el proceso de hidrodestilación. En la tabla 2, se ilustran los rendimientos en el proceso de extracción de los volátiles respecto a los niveles de concentración.

Tabla 2. Recuperación de volátiles por tratamiento

\begin{tabular}{|c|c|c|c|}
\hline Tratamiento & $\begin{array}{c}\text { Masa de extracto }(\mathrm{mg}) \pm \\
\text { desviación estándar }\end{array}$ & $\begin{array}{c}\text { Masa de volátiles recuperados }(\mathrm{mg}) \pm \\
\text { desviación estándar }\end{array}$ & \% volátiles recuperados \\
\hline 1 & $50,4 \pm 0,3$ & $16,8 \pm 0,6$ & 33,4 \\
\hline 2 & $100,3 \pm 0,4$ & $36,9 \pm 2,4$ & 36,7 \\
\hline 3 & $100,3 \pm 0,9$ & $31,5 \pm 1,5$ & 31,4 \\
\hline 4 & $100,4 \pm 0,5$ & $24,7 \pm 0,8$ & 24,6 \\
\hline
\end{tabular}

Como puede apreciarse, se obtuvo mayor cantidad de volátiles para el tratamiento 2, y un significativo porcentaje promedio de IE de 73,12. El posterior proceso de cristalización con hexano permitió obtener IE con una pureza de 97-98\%. Los resultados de los métodos convencionales (Rojano et al., 2007a; García, 2014) reportan la obtención de 1,5 y 1,3\% respectivamente, de isoespintanol a partir de las hojas de O. xylopioides, mientras que por el presente método se obtiene una proporción cercana al 1,2\%. Es importante destacar que los métodos convencionales para la purificación de IE incluyen técnicas de cromatografía en columna y en capa delgada preparativa, con el consiguiente gasto de adsorbentes y disolventes, además del tiempo de operación de estas técnicas, de tal manera que el procedimiento planteado en este estudio representa un ahorro significativo en tiempo e insumos químicos y un método mucho más amigable con el medio ambiente. 
Luego de verificar los supuestos y ejecutar el ANAVA paramétrico de un factor, se encontró que la concentración del extracto de partida tiene un efecto significativo $(p<0,05)$ sobre el rendimiento de los volátiles extraídos. Se encontró a través de la prueba de Tukey, que las concentraciones más bajas del EB en el proceso de hidrodestilación, determinan los mayores contenidos de los volátiles al final del proceso, obteniéndose resultados similares para las concentraciones de 2,27 y 4,54 mg de EB/mL.

\section{Condiciones óptimas del proceso}

El proceso de hidrodestilación se optimizó en $4,54 \mathrm{mg} / \mathrm{mL}$ en un tiempo de 40 minutos en promedio. El flujo en el condensador de $0,5 \mathrm{~mL} / \mathrm{min}$. Para el proceso de extracción se definieron dos extracciones con $10 \mathrm{~mL}$ de diclorometano por un tiempo de $10 \mathrm{~min}$. El proceso de concentración se fijó a $50^{\circ} \mathrm{C}, 1010 \mathrm{mbar}$ y $80 \mathrm{rpm}$. El proceso de cristalización se optimizó con $200 \mu \mathrm{L}$ de hexano y un cristal de $10 \mathrm{mg}$ de IE.

\section{CONCLUSIONES}

Empleando una ruta alternativa se obtuvo isoespintanol a partir del extracto bencínico de O. xylopioides. Se observó que el rendimiento en la extracción de volátiles cambia significativamente, por la influencia de la concentración del extracto. La pureza promedio del compuesto en el hidrodestilado fue de $73,12 \%$ y el rendimiento cercano al $1,2 \%$. Los gastos de insumos y tiempo son sustancialmente bajos, lo que resulta importante si se le compara con el método convencional que emplea cromatografía en columnas sucesivas (de una a tres veces), seguida de cromatografía en capa delgada preparativa. Esto lo hace una técnica atractiva por su rapidez, economía y mínimo efecto ambiental.

\section{AGRADECIMIENTOS}

Los autores agradecen a la Universidad de Córdoba por la financiación del trabajo.

\section{REFERENCIAS}

Angulo, O., Cuca, L.E., y Santafe, G., Aporfinoides en hojas de Oxandra longipetala R. E. Fr. (annonaceae). http://www.redalyc.org/pdf/849/84903304.pdf; ISSN: 0122-1701, Rev. Scientia et Technica, Año XIII, 33, 19$22(2007)$

Arango, G., Cortes, D., y Cavé, A., Three bis-dehydroaporphines from Oxandra cf. major, doi:10.1016/S00319422(00)82394-8, Phytochemistry, 26(4), 1227-1229 (1987a)

Arango, G., Cortes, D., Cassels, B., y Mérienne, C., Azafluorenones from Oxandra cf. major and biogenetic considerations. doi:10.1016/S0031-9422(00)81765-3, Phytochemistry, 26(7), 2093-2098 (1987b)

Arango, N., Vanegas, N., Saez, J., García, C. M., y B. Rojano., Actividad antifúngica del isoespintanol sobre hongos del genero Colletotricum. http://www.redalyc.org/pdf/849/84903375.pdf; ISSN: 0122-1701, Rev. Scientia et Technica Año XIII. (33), 279-280 (2007)

Dade, M., Rojano, B., Tournier, H., y Schinella, G., Apoptosis de células polimorfonucleares humanas por acción del isoespintanol y compuestos relacionados. http://revista.med.unlp.edu.ar/archivos/201010/Dade\% 28FarmacologiaBasica\%29.pdf, Tercera época. Revista de la Facultad de Ciencias Médicas, 2 (1), 1 (2010)

De Sousa, E., Da Silva, A., Cavalheiro, A., Lago, J., y Chaves, M., A new flavonoid derivative from leaves of Oxandra sessiliflora R. E. Fries, doi: 10.5935/0103-5053.20140023, J. Brazilian Chemical Society, 25(4), 704$708(2014 a)$

De Sousa, E., Da Silva, A., Roque, N., Vieira, G., Lago, J., y Chaves, M., Terpenes and steroids from leaves of Oxandra sessiliflora R. E. Fries, doi: 10.1016/j.phytol.2013.09.014, Phytochemistry Letters, 8, 193-195 (2014b)

García, J. Estudio químico, actividad antioxidante y bactericida de los extractos y subextractos de las hojas y corteza de Oxandra xylopioides (Annonaceae). Tesis de Magister, Departamento de Química, Universidad de Córdoba, Montería, Colombia (2014)

Gil, M., y Rojano, B., Inhibición de la polifenoloxidasa extraída del banano (cavendish) por medio de algunos derivados del isoespintanol. http://repository.lasallista.edu.co/dspace/bitstream/10567/148/1/11.\% 20193248.pdf, Corporación Universitaria Lasallista. (2012) 
Hocquemiller, R., Cortés. D., Arango. G.J., Myint. S.H., y Cavé. A., Isolement et synthese de lespintanol, nouveau monoterpene antiparasitaire. doi: 10.1021/np50074a015, J. Nat. Prod., 54(2), 445-442 (1991)

Limón, J., Rodríguez, M., Sánchez, J., Tlapa, D., Metodología bayesiana para la optimización simultánea de múltiples respuestas, doi: 10.4067/S0718-07642012000200017, Inf. Tecnol. 23(2), 151-166, (2012)

Muñoz, D., Cadavid, I., López, J., y Rojano, B., Evaluación genotóxica y citotóxica del isoespintanol en cultivos de linfocitos humanos, doi: http://dx.doi.org/10.15446/abc.v20n1.42529 Acta Biológica Colombiana, 13(3), 71 (2008)

Murillo, J., Las Annonaceae de Colombia, http://www.redalyc.org/pdf/491/49120104.pdf; ISSN: 0124-5376, Rev. Biota Colombiana, 2, 49 - 58 (2001)

Pino, J., Leaf oil of Oxandra lanceolata (Sw.) Baill. from Cuba, doi: 10.1080/10412905.2005.9698997, J. of Essential Oil Research, 17(5), 567-568 (2005)

Rates, S., Plants as source of drugs. (Review), doi: 10.1016/S0041-0101(00)00154-9, Toxicon, 39(5), 603$613(2001)$

Rojano, B., Pérez, E., Figadére, B., Martin, M., Recio, M., Giner, R., y Sáez, J., Constituents of Oxandra cf. xylopioides with Anti-inflammatory Activity, doi: 10.1021/np060333v, J. Nat. Prod., 70(5), 835-838 (2007a)

Rojano, B., Gaviria, C., Sáez, J., Yepes, F., Muñoz, F., y Ossa, F., Berenjenol aislado de Oxandra cf xylopioides (annonaceae) como insecticida, Vitae, 14 (2), 95-100 (2007b)

Rojano, B., Gaviria, C., Gil, M., Sáez, J., Schinella, G., y Tournier, H., Actividad antioxidante del isoespintanol en diferentes medios, Vitae, 15(1), 173-181 (2008a)

Rojano B, Saez, J., Schinella, G., Quijano, J., Vélez, E., Gil, A., Notario, R., Experimental and theoretical determination of the antioxidant properties of isoespintanol (2-isopropyl-3,6-dimethoxy-5-methylphenol). doi:10.1016/j.molstruc.2007.07.010, J. Mol. Struct., 877, 1-6 (2008b)

Rojano, B., Montoya, S., Yépez, F., y Sáez, J., Evaluación de isoespintanol aislado de Oxandra cf. xylopioides (Annonaceae) sobre Spodoptera frugiperda J.E. Smith (Lepidoptera: Noctuidae). http://www.scielo.org.co/pdf/rfnam/v60n1/a05v60n1.pdf, Rev. Fac. Nal. Agr. Medellín, 60(1), 3691-3702 (2007c)

Tinto, W., y Blair, L., Terpenoid constituents of Oxandra asbeckii. doi: 10.1021/np50084a001, J. Nat. Prod., 55(6), 701-706 (1992)

Zhang, J., El Shabrawy, A-R., M. Shanawany, El., Schiff, P., y Slatkin, D., New azafluorene alkaloids from Oxandra xylopioides. doi: 10.1021/np50053a005, J. Nat. Prod., 50(5), 800-806 (1987) 